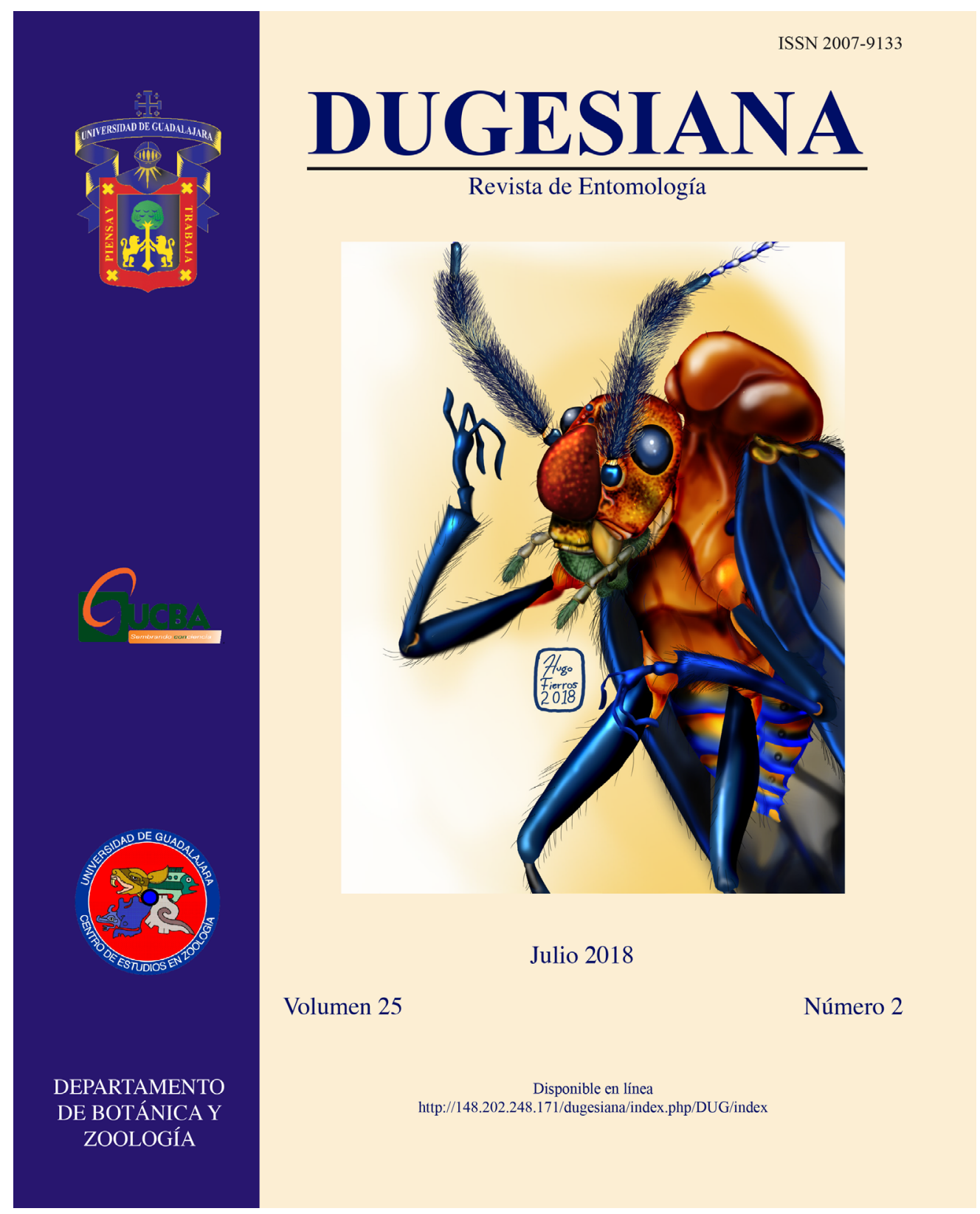

Dugesiana, Año 25, No. 2, julio 2018-diciembre 2018 (segundo semestre de 2018), es una publicación semestral, editada por la Universidad de Guadalajara, a través del Centro de Estudios en Zoología, por el Centro Universitario de Ciencias Biológicas y Agropecuarias. Camino Ramón Padilla Sánchez \# 2100, Nextipac, Zapopan, Jalisco, Tel. 37771150 ext. 33218, http://148.202.248.171/dugesiana/index.php/DUG/index, glenusmx@gmail.com. Editor responsable: José Luis Navarrete Heredia. Reserva de Derechos al Uso Exclusivo 04-2009-062310115100203, ISSN: 2007-9133, otorgados por el Instituto Nacional del Derecho de Autor. Responsable de la última actualización de este número: José Luis Navarrete Heredia, Editor y Ana Laura González-Hernández, Asistente Editorial. Fecha de la última modificación 25 de julio 2018, con un tiraje de un ejemplar.

Las opiniones expresadas por los autores no necesariamente reflejan la postura del editor de la publicación.

Queda estrictamente prohibida la reproducción total o parcial de los contenidos e imágenes de la publicación sin previa autorización de la Universidad de Guadalajara. 


\title{
Sinopsis de las especies de Mantodea en México con nuevos registros de distribución para Chiapas (Insecta: Dictyoptera)
}

\section{Synopsis of Mantodea species in Mexico with new distribution records for Chiapas (Insecta: Dictyoptera)}

\section{Erick Hernández-Baltazar ${ }^{1, *}$, Benigno Gómez² y Ana Iris Melgar-Martínez ${ }^{1}$}

${ }^{1}$ Instituto de Ciencias Biológicas, Universidad de Ciencias y Artes de Chiapas. Tuxtla Gutiérrez, Chiapas, México. *E-mail: kcireherbal@gmail.com; ${ }^{2}$ Departamento de Conservación de la Biodiversidad, El Colegio de la Frontera Sur. San Cristóbal de Las Casas, Chiapas, México.

\section{RESUMEN}

Se actualiza la lista de distribución estatal de los mántidos de México, registrando 72 especies para el país. Además, se presentan nuevos registros de mántidos para el estado de Chiapas, basado en colectas realizadas en 2015 en el Centro Ecoturístico "Santuario de Cocodrilo, Tres Lagunas" en la Selva Lacandona y se documenta nuevos datos de distribución para Liturgusa maya Saussure \& Zehntner, 1894.

Palabras claves: Distribución, mantis, Neotropical, Selva Lacandona.

\begin{abstract}
The distribution of the mantids of Mexico is updated, recording 72 species for the country. In addition, new records of mantids for the state of Chiapas are presented, based on fieldwork performed in 2015 at the Ecotourism Center "Santuario de Cocodrilo, Tres Lagunas" in the Lacandona Forest, furthermore new distribution data are documented for Liturgusa maya Saussure \& Zehntner, 1894.

Key words: Distribution, mantis, Neotropical, Lacandona Forest.
\end{abstract}

Los mántidos son un orden de Dictyoptera que comprende aproximadamente 2500 especies a nivel mundial (Patel et al. 2016, Patel y Singh 2016, Roy 2014). En la región neotropical los mántidos alcanzan cerca de 498 especies (Rivera 2010) y están distribuidos dentro de sus diferentes ecosistemas terrestres, desde el nivel del mar hasta cerca de los $3000 \mathrm{~m}$ s.n.m. (Agudelo et al. 2007, Ehrmann y Koçak 2009, Rivera 2010). La diversidad de mántidos en México ha sido escasamente documentada, registrándose 71 especies (distribuidas en 24 géneros) y la misma carencia de conocimiento de este taxa ocurre para Chiapas, donde se han registrado solo seis especies (Hernández-Baltazar y Gómez 2017, Rehn 1935a, 1935b, Rodrigues et al. 2017, Roy 2012, Svenson 2014, Terra 1995).

\section{MATERIAL Y MÉTODOS}

Considerando la recomendación de Mariño-Pedraza (2011) quien indica que la presencia de ninfas y adultos de mántidos ocurre en primavera y verano, se realizaron colectas durante los meses de lluvia de julio y agosto de 2015 en las inmediaciones del Centro Ecoturístico "Santuario de Cocodrilo, Tres Lagunas" (Figura 1), en la Selva Lacandona (Ocosingo, Chiapas), utilizando trampas de luz y colecta directa manual, los cuales son considerados métodos idóneos y con mayor posibilidad de captura (Agudelo y Chica 2003). Los ejemplares capturados se colocaron en viales con alcohol al 70\%, transportándose posteriormente al laboratorio para su procesamiento curatorial, identificación taxonómica y extracción de genitalia (Agudelo y Chica 2002, Ariza et al. 2012, Arteaga et al. 2014, Brannoch et al. 2017, Giglio-Tos 1927, Klass 1997, Márquez-Luna 2005, Ortega y Márquez 1987, Romero y Nelson 2008, Svenson 2014). Los especímenes se revisaron taxonómicamente, ubicándolos categóricamente mediante las propuestas de Rivera y Svenson (2016) para la superfamilia Acanthopoidea y para familias y subfamilias de Mantodea se ocupó lo indicado por Wieland y Schütte (2017). La distribución documentada de los ejemplares se consultó en los trabajos de Agudelo et al. (2007), Battiston et al. (2005), Battiston y Picciau (2008), Cano-Santana y Ramos-Rivera (2017), Ehrmann (2002), Giglio-Tos (1927), Hebard (1922, 1932), Hernández-Baltazar y Gómez (2017), Jantsch (1999), Kirby (1904), Lombardo y Agabiti (2001), Mantodea Image Database [MantID] (2018), Marshall (1975), Maxwell (2014), Pfäuti y Hollier (2012), Rehn (1935a), Saussure y Zehntner (1894), Svenson (2014), Svenson et al. (2016), Terra (1995) y Werner (1925). Todo el material colectado se encuentra depositado en la colección de El Colegio de La Frontera Sur, Unidad San Cristóbal, Chiapas (ECO-SC-E).

RESULTADOS
Para Chiapas se capturaron cinco ejemplares de


mántidos, pertenecientes a tres familias, tres géneros y tres especies, que ha permitido conocer mejor su mantidofauna (véase abajo). Los resultados indican que el estado es una región de México que puede albergar muchas más especies que aún pasan desapercibidas en sus variados ecosistemas.

\section{Datos nuevos}

Familia Mantidae

Subfamilia Stagmomantinae

Stagmomantis venusta Saussure \& Zehntner, 1894 (Figura 2A-B)

Distribución: Centro América, México (Oaxaca).

Material examinado: Santuario de Cocodrilo, Tres Lagunas; lat $16^{\circ} 50^{\prime} 31.1^{\prime \prime} \mathrm{N}$, long $91^{\circ} 08^{\prime} 52.8^{\prime \prime} \mathrm{W}, 371$ m; 01 adulto $\bigcirc ;$ 26.VII.2015; E. Hernández-B, A.I Melgar cols. (ECO-SC-E-68123).

\section{Superfamilia Acanthopoidea Familia Liturgusidae Subfamilia Liturgusinae}

Liturgusa maya Saussure \& Zehntner, 1894 (Figura 3A-D)

Distribución: Colombia, Costa Rica, Ecuador, Guatemala, Honduras, México (Chiapas, Guerrero, Quintana Roo, Tabasco, Veracruz, Yucatán), Nicaragua, Panamá, Perú y Venezuela.

Material examinado: Santuario de Cocodrilo, Tres Lagunas; lat $16^{\circ} 50^{\prime} 37.2^{\prime \prime} \mathrm{N}$, long $91^{\circ} 08^{\prime} 46.3^{\prime \prime} \mathrm{W}, 360$ m; 01 adulto $\delta$ y 2 ninfas; 22.VII.2015, 26.VII.2015; E. Hernández-B, A.I Melgar cols. (ECO-SC-E-68127).

\section{Superfamilia Acanthopoidea Familia Thespidae}

Subfamilia Pseudomiopteryginae

Pseudomiopteryx infuscata Saussure \& Zehntner, 1894 (Figura 4A-D)

Distribución: Bolivia, Brasil, Costa Rica, Guatemala, México (Veracruz), Nicaragua, Panamá y Venezuela.

Material examinado: Santuario de Cocodrilo, Tres Lagunas; lat $16^{\circ} 50^{\prime} 36.4^{\prime \prime} \mathrm{N}$, long $91^{\circ} 08^{\prime} 43.9^{\prime \prime} \mathrm{W}, 387$

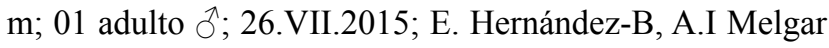
cols. (ECO-SC-E-68126).

Además de estos resultados, se incluye datos adicionales que fueron omitidos en la lista conocida previamente para el territorio nacional (véase Hernández-Baltazar y Gómez 2017), siendo los siguientes (Registro omitido para México señalado por un asterisco $\left[^{*}\right]$ y registros estatales omitidos marcados por doble asterisco $\left[{ }^{* *}\right]$ ):

Familia Mantidae

Subfamilia Choeradodinae

Género Choeradodis Audinet-Serville, 1831

C. rhomboidea Stoll, 1813: Veracruz** (Jantsch 1999)

Subfamilia Stagmomantinae

Género Stagmomantis Saussure, 1869
S. carolina (Johansson, 1763): Chiapas, Jalisco, Morelos**, Oaxaca**, Sinaloa, Veracruz, Yucatán (HernándezBaltazar y Gómez 2017, Werner $1925^{* *}$ )

S. heterogamia Saussure \& Zehntner, 1894: Baja California**, Oaxaca (Hernández-Baltazar y Gómez 2017, Jantsch 1999**)

S. montana Saussure \& Zehntner, 1894: Chiapas**, Guerrero, Jalisco, Oaxaca, Veracruz (HernándezBaltazar y Gómez 2017, Jantsch 1999**)

S. parvidentata Beier, 1931: Chiapas** (Jantsch 1999)

S. theophila Rehn, 1904: Chiapas** (Jantsch 1999)

S. vicina Saussure, 1870: Chiapas**, Oaxaca, Veracruz** (Hernández-Baltazar y Gómez 2017, Jantsch 1999**)

\section{Subfamilia Vatinae}

Género Pseudovates Saussure, 1869

P. arizonae Hebard, 1935: Guerrero* (Svenson et al. 2016)

P. chlorophaea (Blanchard, 1836): Nuevo León, San Luis Potosi**, Sinaloa, Tabasco, Tamaulipas**, Veracruz, Yucatán (Hernández-Baltazar y Gómez 2017, Svenson et al. 2016**)

P. cornuta (Saussure \& Zehntner, 1894): Jalisco, Tamaulipas**, Veracruz (Hernández-Baltazar y Gómez 2017, Svenson et al. 2016**)

P. tolteca (Saussure, 1859): Ciudad de México, Veracruz** (MantID 2018**, Svenson et al. 2016)

\section{Género Vates Burmeister, 1838}

V. chopardi (Deeleman-Reinhold, 1957): Nayarit**,

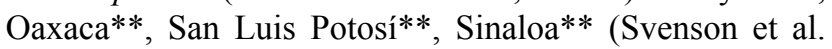
2016)

Género Zoolea Audinet-Serville, 1839

Z. orba Burmeister, 1838: Chiapas** (Jantsch 1999)

Superfamilia Acanthopoidea

Familia Liturgusidae

Subfamilia Liturgusinae

Género Liturgusa Saussure, 1869

L. maya Saussure \& Zehntner, 1894: Chiapas, Guerrero, Jalisco**, Quintana Roo, Tabasco, Veracruz, Yucatán (Hernández-Baltazar y Gómez, 2017, MantID 2018**)

\section{Familia Thespidae \\ Subfamilia Thespinae \\ Género Oligonyx Saussure, 1869}

O. bicornis Saussure, 1869: Oaxaca**, Veracruz (Hernández-Baltazar y Gómez 2017, Pfäuti y Hollier $2012 * *)$

\section{Género Pseudomusonia Werner, 1909 \\ P. lineativentris (Stål, 1877): Nuevo León** (Jantsch 1999)}

\section{DISCUSIÓN}

Las especies consideradas como nuevos registros para el estado de Chiapas son Pseudomiopteryx infuscata y Stagmomantis venusta. P. infuscata se ha registrado para 
la vertiente del Golfo, encontrándose datos de colecta en Córdova, Veracruz (Kirby 1904, Marshall 1975, Saussure y Zehntner 1894). Con este nuevo hallazgo en la Selva Lacandona, se amplía la distribución de esta especie 650 $\mathrm{km}$ al sureste del territorio nacional. En el caso de $S$. venusta existen registros solamente en el estado de Oaxaca (Cano-Santana y Ramos-Rivera 2017). No obstante, al ser Stagmomantis un género principalmente Neotropical, distribuyéndose en Estados Unidos, México, Venezuela, Colombia, Ecuador y Perú (Agudelo et al. 2007, Maxwell 2014), no es de extrañarse haberlo encontrado en Chiapas. Con el registro en la Selva Lacandona, la especie amplía su distribución en México cerca de 590 km al sur (Oaxaca).

Liturgusa es un género sudamericano con penetración en Centroamérica (Saussure y Zehntner 1894, Svenson 2014), y encuentra su distribución más septentrional en tierras mexicanas. L. maya se ha registrado en Chiapas, solo en la vertiente del Pacifico, siendo Tapachula el único dato disponible (Svenson 2014). No obstante, existen registros para otros estados de la vertiente del Golfo: Guerrero, Quintana Roo, Tabasco, Veracruz y Yucatán (Battiston y Picciau 2008, Hebard 1932, Rehn 1935a, Svenson 2014), por lo que no es raro haberla encontrado en la Selva Lacandona. Su registro más próximo es la localidad de Teapa, Tabasco (Battiston y Picciau 2008, Svenson 2014) a unos $200 \mathrm{~km}$ al noroeste de la localidad de colecta (Santuario de Cocodrilo).

La revisión de la literatura permitió el registro para México de Pseudovates arizonae Hebard, 1935. Con la inclusión de esta especie a la lista presentada por Hernández-Baltazar y Gómez (2017) se actualiza el número de registros para el país, pasando de 71 a 72 especies. $P$. arizonae se localiza en él estado de Guerrero (Svenson et al. 2016).

Por último, se actualiza el número de registros estatales de Hernández-Baltazar y Gómez (2017), incluyendo a Baja California con un registro y aumentando la riqueza de especies documentadas para Chiapas (13), Guerrero (10), Jalisco (14), Morelos (7), Nayarit (4), Nuevo León (5), Oaxaca (18), San Luis Potosí (6), Sinaloa (22), Tamaulipas (5) y Veracruz (20).

\section{AGRADECIMIENTOS}

Los autores agradecen en especial a Pablo Chankin Najbor, presidente del Centro Ecoturístico "Santuario de Cocodrilo, Tres Lagunas" por las facilidades otorgadas para la realización de este estudio y por su amable hospitalidad durante la estancia en el lugar de colecta. A la lic. Carla Gasca Suárez, por la revisión crítica y corrección del manuscrito. Al M. en C. Jorge A. Mérida Rivas por apoyo técnico y toma de fotografías.

\section{LITERATURA CITADA}

Agudelo, A.A. y L.M. Chica (Eds). 2002. Mántidos: introducción al conocimiento del orden Mantodea. Universidad Distrital Francisco José de Caldas, Bogotá,
Colombia.

Agudelo, A.A. y L.M. Chica. 2003. Mántidos de la Orinoquia colombiana: contribución al conocimiento de su diversidad genérica y algunos aspectos bioecológicos (Insecta: Mantodea). Revista Colombiana de Entomología, 29(2): 127-136.

Agudelo, A.A., F. Lombardo and L.J. Jantsch. 2007. Checklist of the neotropical mantids (Insecta, Dictyoptera, Mantodea). Biota Colombiana, 8(2): 105158.

Ariza, G.M., J.A. Salazar y N.A. Canal. 2012. Especies y distribución de los mántidos (Mantodea) del departamento del Tolima, Colombia. Revista Colombiana de Entomología, 38(2): 282-290.

Arteaga, L.A., A.C. de La Parra, M.C. Medellín y N.J. Martínez. 2014. Mantidofauna (Insecta: Mantodea) en fragmentos de Bosque Seco Tropical (Bs-T) en el Departamento del Atlántico (Colombia). Boletín Científico. Centro de Museos. Museo de Historia Natural, 18(2): 243-262.

Battiston, R., P. Fontana, B. Agabiti and P.L. García-García. 2005. Mantodea collected in Mexico during an $8800 \mathrm{~km}$ orthopterological trip (Insecta: Mantodea). Accademia Roveretana degli Agiati, Atti, 8(5): 199-215.

Battiston, R. and L. Picciau. 2008. Contribution to the knowledge of the Mantodea of Ecuador with the description of the male of Pseudoxyops perpulchra (Westwood, 1889) (Mantodea: Mantidae: Stagmatopterinae). Biodiversity of South America, I. Memoirs on Biodiversity, 1: 19-30.

Brannoch, S.K., F. Wieland, J. Rivera, K.-D. Klass, O. Béthoux and G.J. Svenson. 2017. Manual of praying mantis morphology, nomenclature, and practices (Insecta, Mantodea). ZooKeys, 696: 1-100.

Cano-Santana, Z. y P. Ramos-Rivera. 2017. Ortopteroides de Oaxaca, México: Orthoptera, Mantodea y Phasmatodea. Comisión Nacional para el Conocimiento y Uso de la Biodiversidad. http://www.gbif.org/dataset/255d10275e6f-443c-910b-b9f405e2f14d. Fecha de consulta: 15 de octubre de 2017.

Chopard, L. 1916. Étude des Mantides Américains. Annales de la Société entomologique de France, 85: 173-174.

Ehrmann, R (Ed). 2002. Mantodea. Gottesanbeterinnen der Welt. Natur und Tier Verlag GmbH, Münster.

Ehrmann, R. and A.Ö Koçak. 2009. The neotropical mantids (Insecta: Dictyoptera: Mantodea). Cesa News, 49: 1-18.

Giglio-Tos, E. 1927. Orthoptera Mantidae. (pp. 1-707). En: Schulze F. E. und W. Kükenthal (Eds.). Das Tierreich, eine Zusammenstellung und Kennzeichnung der rezenten Tierformen. Fascicle: 50. De Gruyter \& Co., Berlín, Alemania.

Hebard, M. 1922. Studies in the Mantidae and Phasmidae of Panama (Orthoptera). Transactions of the American Entomological Society, 48(4): 327-362.

Hebard, M. 1932. New species and records of Mexican Orthoptera. Transactions of the American Entomological 
Society, 58: 201-371.

Hernández-Baltazar, E. y B. Gómez. 2017. Distribución estatal de mántidos en México (Dictyoptera: Mantodea). Boletín de la Sociedad Entomológica Aragonesa (S. E. A.), 61: 175-178.

Jantsch, L.J. 1999. Estudos filogenéticos em Mantódeos americanos (Insecta; Pterygota; Mantodea). Tesis de doctorado, PUCRS, Porto Alegre.

Kirby, W.F. 1904. A synonymic catalogue of Orthoptera: Orthoptera, Euplexoptera, Cursoria et Gressoria. Bulletin of the British Museum (Natural History) Entomology, (1): 207-316.

Klass, K.-D. 1997. The external male genitalia and the phylogeny of Blattaria and Mantodea. Bonner Zoologische Monographien, 42: 1-341.

Lombardo, F. and B. Agabiti. 2001. The mantids from Ecuador, with some biogeographic considerations. Journal of Orthoptera Research, 10(1): 89-104.

Mantodea Image Database (MantID). 2018. https:// specimens.mantodearesearch.com/. Fecha de consulta: 10 de enero de 2018.

Mariño-Pedraza, E. 2011. Los mántidos. Biodiversitas, 95: 12-16.

Márquez-Luna, J. 2005. Técnicas de colecta y preservación de insectos. Boletín de la Sociedad Entomológica Aragonesa (S. E. A.), 37: 385-408.

Marshall, J.A. 1975. A catalogue of the primary types of Mantodea (Dictyoptera) in the British Museum (Natural History). Bulletin of the British Museum (Natural History) Entomology, 31(8): 317-318.

Maxwell, M.R. 2014. A synoptic review of the genus Stagmomantis (Mantodea: Mantidae). Zootaxa, 3765(6): 501-525.

Ortega, G. y C. Márquez. 1987. Ortópteros de la Estación de Biología "Chamela", Jalisco (Insecta: Orthoptera). Anales del Instituto de Biología, Universidad Nacional Autónoma de México (Serie Zoología), 58(1): 35-62.

Patel, S. and R. Singh. 2016. Updated checklist and global diversity of Chaeteessidae, Mantoididae, Metallyticidae, Acanthopidae, Amorphoscelididae and Sibyllidae (Mantodea: Insecta). International Journal of Research Studies in Zoology, 4(2): 55-67.

Patel, S., G. Singh and R. Singh. 2016. A checklist of global distribution of Liturgusidae and Thespidae (Mantodea: Dictyoptera). Journal of Entomology and Zoology Studies, 4(6): 793-803.

Pfäuti, P. and J. Hollier. 2012. Additions to the catalogue of mantid type material deposited in the Muséum d'histoire naturelle de Genéve (Insecta: Mantodea). Revue suisse de Zoologie, 119(3): 261-267.

Recibido: 28 de febrero 2018

Aceptdo: 7 de junio 2018
Rehn, J.A.G. 1935a. The Orthoptera of Costa Rica. Part 1: Mantidae. Proceedings of the Academy of Natural Sciences of Philadelphia, 87: 167-272.

Rehn, J.A.G. 1935b. On certain Mexican and Central American species of Melliera and Stagmomantis (Orthoptera: Mantidae). Transactions of the American Entomological Society, 61(4): 317-329.

Rivera, J. 2010. A historical review of praying mantid taxonomy and systematics in the neotropical region: state of knowledge and recent advances (Insecta: Mantodea). Zootaxa, 2638: 44-64.

Rivera, J. and G.J. Svenson. 2016. The Neotropical “polymorphic earless praying mantises"-Part I: molecular phylogeny and revised higher-level systematics (Insecta: Mantodea, Acanthopoidea). Systematic Entomology, 41: 607-649.

Rodrigues, H.M., J. Rivera, N. Reid and G.J. Svenson. 2017. An elusive neotropical giant, Hondurantemna chespiritoi gen. n. \& sp. n. (Antemninae, Mantidae): a new lineage of mantises exhibiting an ontogenetic change in cryptic strategy. ZooKeys, 680: 73-104.

Romero, R. y F. Nelson. 2008. Guía ilustrada de mántidos comunes de Colombia. Bogotá, Colombia.

Roy, R. 2012. Une curieuse ressemblance entre des espèces des genres voisins Zoolea

Audinet Serville, 1838, et Vates Burmeister, 1838 (Dict., Mantidae, Vatinae). Bulletin de la Société entomologique de France, 117(3): 380.

Roy, R. 2014. A historical review of nomenclature and highlevel classification of praying mantises (Mantodea), including a provisional checklist of the names associated to suprageneric ranks. Zootaxa, 3797: 9-28.

Saussure, H. et L. Zehntner. 1894. Biologia centraliamericana. Insecta. Orthoptera. Fam. Mantidae. Société entomologique Zurich, (1): 123-197.

Svenson, G.J. 2014. Revision of the neotropical bark mantis genus Liturgusa Saussure, 1869 (Insecta, Mantodea, Liturgusini). ZooKeys, 390: 1-214.

Svenson, G.J., C. Medellín and C. Sarmiento. 2016. Reevolution of a morphological precursor of crypsis investment in the newly revised horned praying mantises (Insecta, Mantodea, Vatinae). Systematic Entomology, 41(1): 229-255.

Terra, P.S. 1995. Revisão sistemática dos gêneros de louvaa-deus da região neotropical. Revista Brasileira de Entomología, 39(1): 13-94.

Werner, V.F. 1925. Zur Kenntnis amerikanischer Mantodeen (Orthoptera Oothecaria). Konowia, Ztschr. f. syst. Insektenkde, (4): 160-168.

Wieland, F. und K. Schütte. 2017. Fortschritte und Perspektiven in der Erforschung der Evolution und Phylogenie der Mantodea (Insecta: Dictyoptera). Entomologie heute, 29: 1-23. 


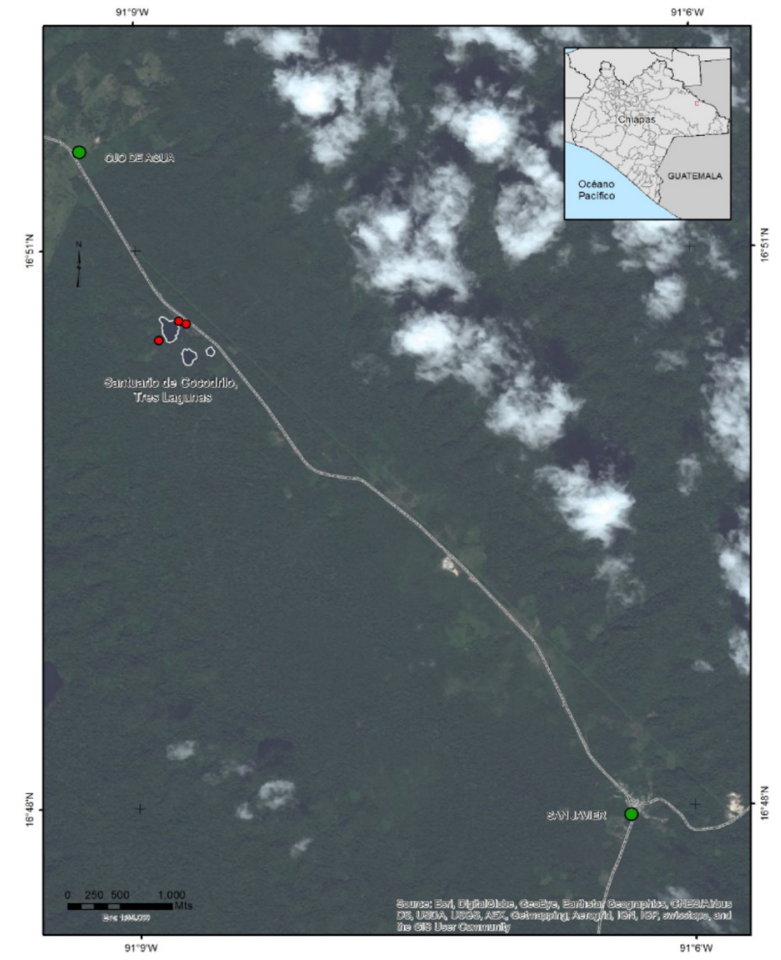

Figura 1. Mapa de ubicación del Centro Ecoturístico "Santuario de Cocodrilo, Tres Lagunas".

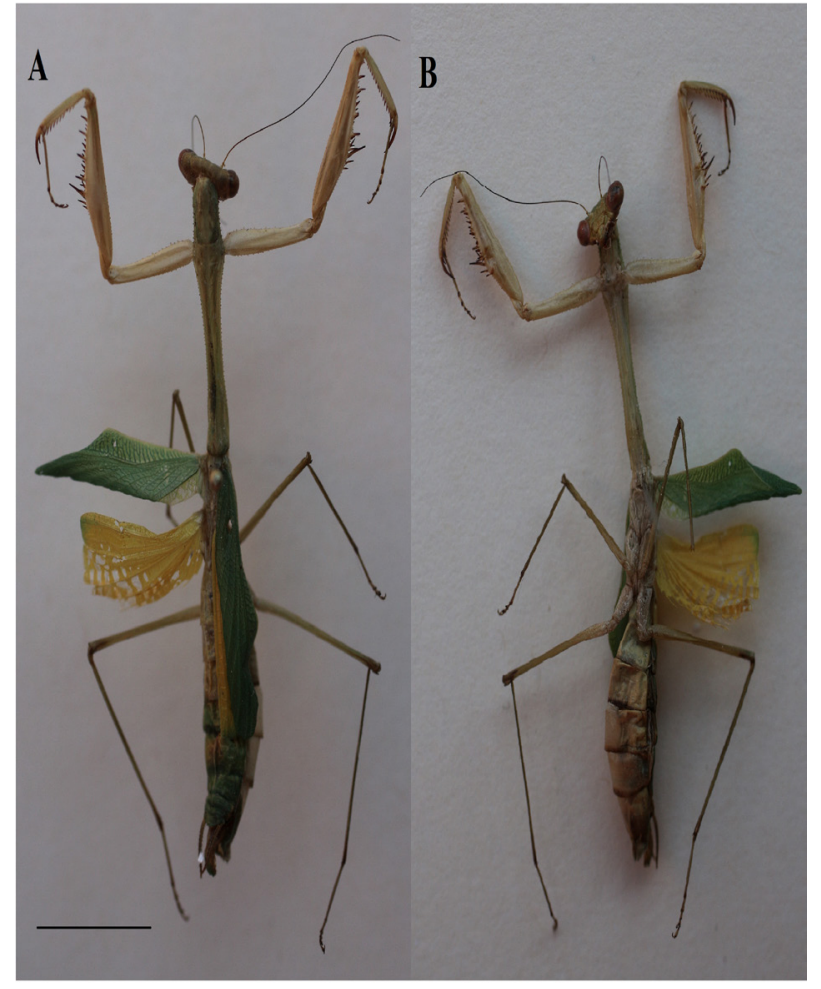

Figura 2. Hembra de Stagmomantis venusta. A) Hábito dorsal, B) Hábito ventral (barra de escala= $10 \mathrm{~mm}$ ).

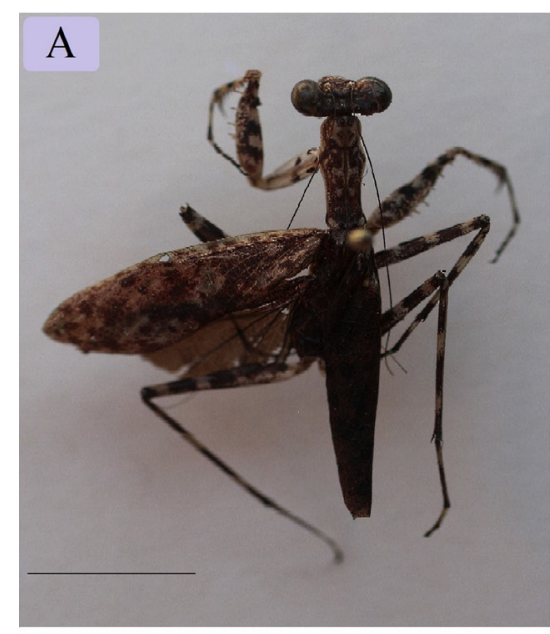

$\mathrm{C}$

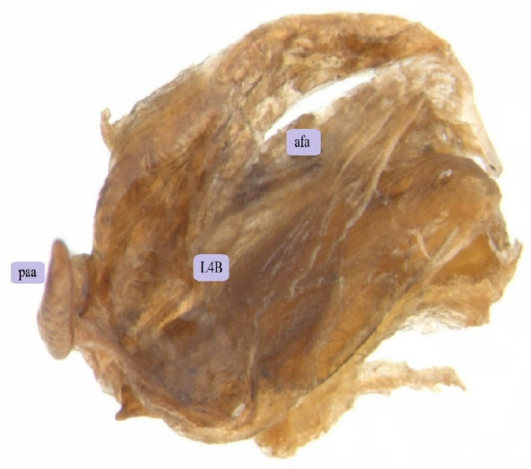

$\mathrm{B}$

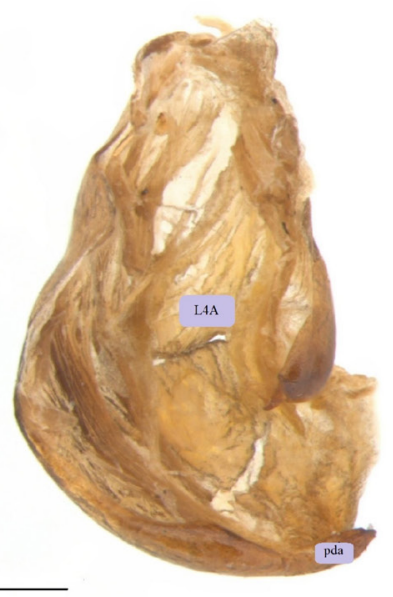

$\mathrm{D}$

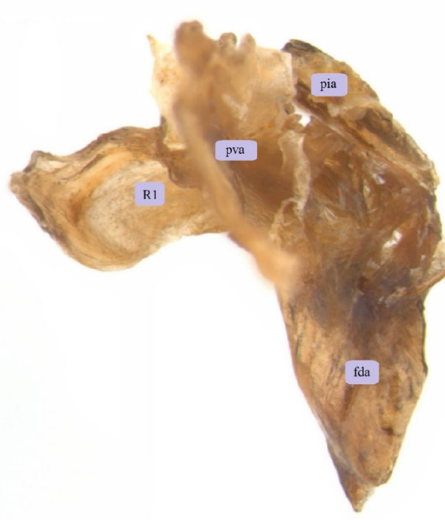

Figura 3. Lámina de Liturgusa maya. A) Hábito dorsal (barra de escala $=5 \mathrm{~mm}$ ). Complejo fálico: B) Falómero ventral, C) Falómero izquierdo, D) Falómero derecho (barra de escala $=1 \mathrm{~mm}$ ). Abreviaturas: L4A: Falómero ventral, L4B: Falómero izquierdo, R1: Falómero derecho, pda: Proyección distal, afa: Apófisis faloidal, paa: Proceso apical, pia: Placa ventral, pva: Proceso ventral, fda: Primer esclerito. 

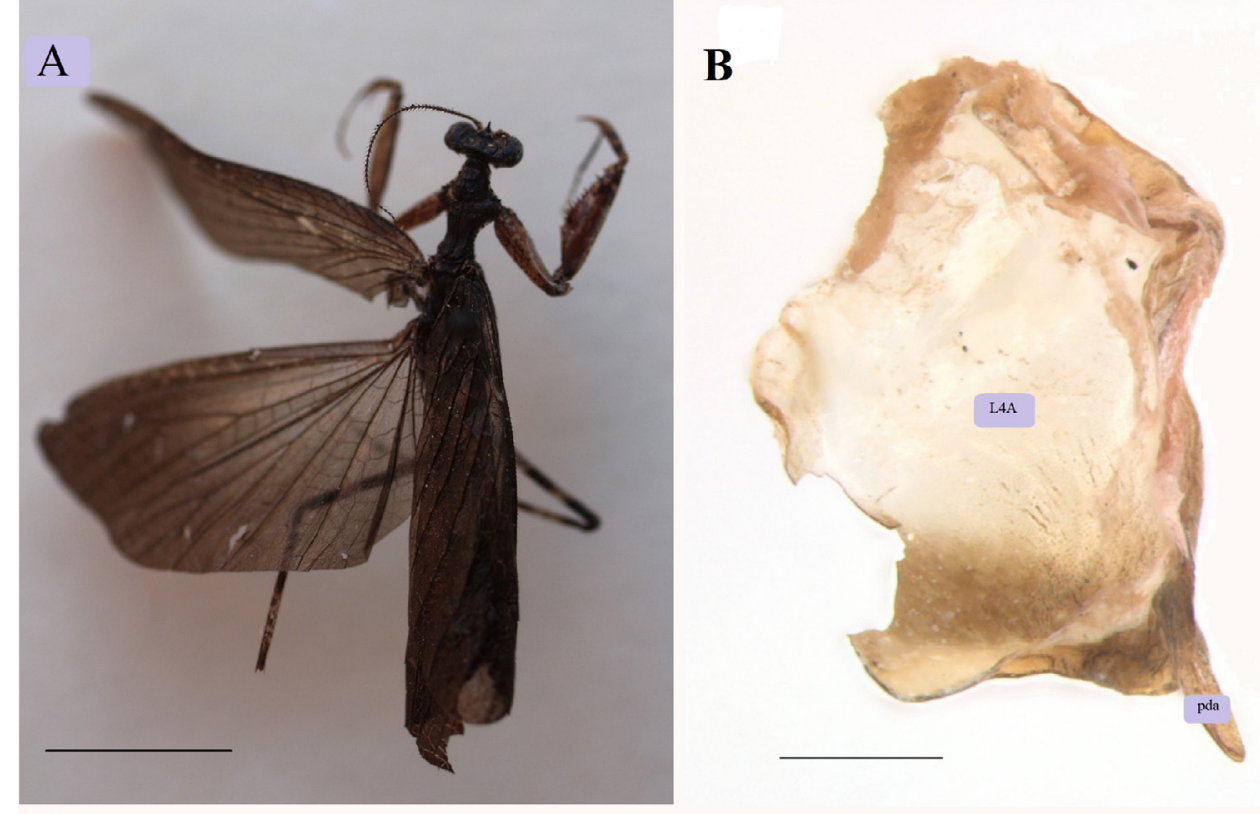

\section{C}

\section{D}
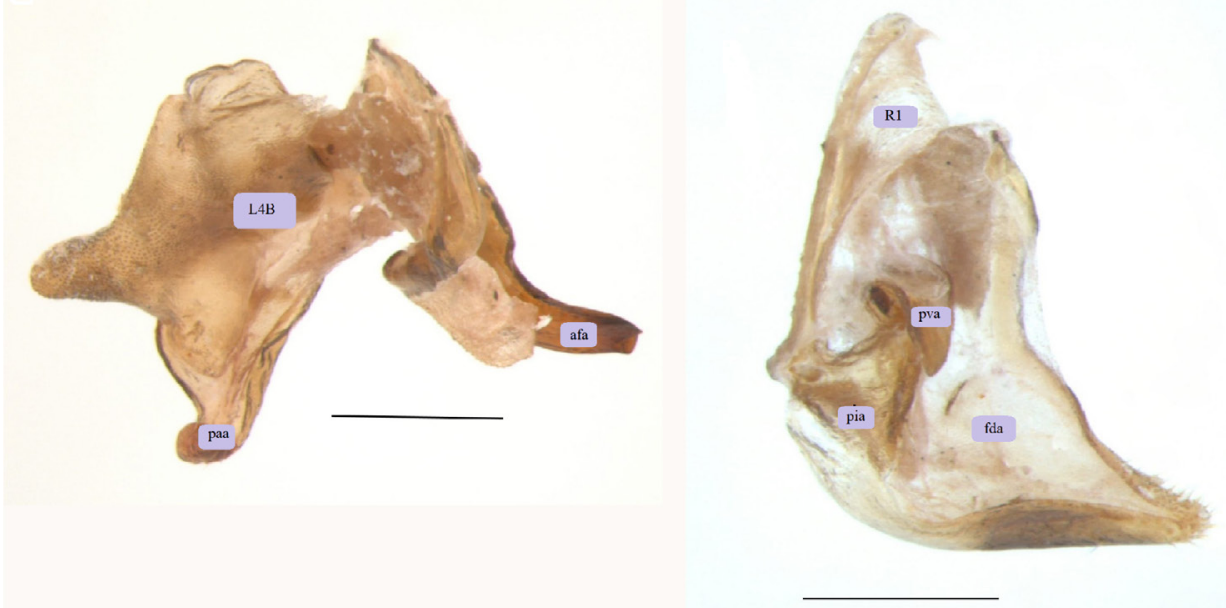

Figura 4. Lámina de Pseudomiopteryx infuscata. A) Hábito dorsal (barra de escala $=5 \mathrm{~mm}$ ). Complejo fálico: B) Falómero ventral, C) Falómero izquierdo, D) Falómero derecho (barra de escala=1 mm). Abreviaturas: L4A: Falómero ventral, L4B: Falómero izquierdo, R1: Falómero derecho, pda: Proyección distal, afa: Apófisis faloidal, paa: Proceso apical, pia: Placa ventral, pva: Proceso ventral, fda: Primer esclerito. 